\title{
KINEMATICS OF THE GALACTIC BULGE: THE VELOCITY ELLIPSOID
}

\author{
Dante Minniti \\ Steward Observatory, Univ. of Arizona, Tucson AZ 85721, U.S.A.
}

Abstract: We investigate the shape of the velocity ellipsoid in the Galactic bulge by combining radial velocities and proper motions. We find that the velocity ellipsoid is nearly isotropic.

The Galactic bulge is the only one where we can actually measure the 3-D kinematics. Because of the lack of 3-D information in distant elliptical galaxies and bulges of spirals, the degree of anisotropy has been just an assumption in every kinematic study of bulges and elliptical galaxies (e.g. Binney and Tremaine 1987, 'Galactic Dynamics', Princeton Univ. Press). With accurate determinations of proper motions towards different bulge fields, we would be in position to constrain that parameter for the first time.

Cudworth (1986, A.J. 92, 348, referred to as C86) measured proper motions in the field of the globular cluster M22 $(l=9.9, b=-7.6)$. The foreground contamination by bulge stars is important in this low latitude field at a projected distance of $1.6 \mathrm{kpc}$ from the Galactic center, as can be seen in C86 color magnitude diagrams. About 200 stars were found to be nonmembers of M22 on the basis of proper motions. By doing appropriate color and magnitude cuts, we select 81 of them which lie in the region of the CMD occupied by the bulge giant branch. Minniti et al. (1992, Ap.J. 393, L47) have measured radial velocities for 78 spectroscopically confirmed $\mathrm{K}$ giants in a field at $l=8, b=7$, most of which are expected to be bulge members. This field lies in a different quadrant, but at about the same projected distance from the Galactic center as the M22 field. Thus, it is not unreasonable to combine the two data sets to study the 3-D kinematics of the bulge. None of our conclusions rely on the absolute proper motions, we focus here on the relative proper motions, which give the dispersions $\sigma_{l}$ and $\sigma_{b}$.

The proper motions reveal that the velocity ellipsoid projected on the plane of the sky is nearly isotropic, with slight elongation towards the Galactic center. If we assume the distance to the Galactic center to be $\mathrm{R}_{0}=7.5 \mathrm{kpc}$, both the radial velocity dispersion $\sigma_{r}$ and the two proper motion dispersions $\sigma_{l}$ and $\sigma_{b}$ are similar, consistent with isotropy. The Figure below shows the distribution of radial velocities and proper motions in the $1, \mathrm{~b}$ directions, scaled assuming $\mathrm{R}_{0}=7.5 \mathrm{kpc}$. Recent work by Spaenhauer et al. (1992, A.J. 103, 297) in Baade's window shows agreement with our results. 
We divide the proper motion sample into 2 subsamples with colors redder and bluer than $(\mathrm{B}-\mathrm{V})_{0}=1.4$, corresponding roughly to metal rich and metal poor giants, respectively. Such a division is preliminary, and must be confirmed by more accurate metallicity determinations. However, we find that the metal rich stars tend to have smaller $\sigma_{l}$ and $\sigma_{b}$ than the metal poor stars. The radial velocities show the same effect, the metal poor subsample has a larger velocity dispersion than the metal rich one. Also, the metal rich stars have a shift in the mean $\mu_{l}$ with respect to the metal poor stars. This shift is in the direction of galactic rotation, again evidence that the metal rich subsample is rotating more rapidly. It is important to note that these effects are small (only $\sim 2 \sigma$ ), and their reality should be checked with more extensive data samples.

To analyze the data, Minniti \& White (1993, in preparation) derive the geometry involved in the cases with spherical and cylindrical symmetry. We model the bulge density and velocity dispersion laws, and perform Monte-Carlo simulations to reproduce the data. We find that a new and independent measure of the distance to the Galactic center can be obtained from combining the proper motions with radial velocities. A distance of $R_{0}=7-8 \mathrm{kpc}$ is found from the $C 86$ proper motions, the uncertainty is given only by the small size of the sample.

In summary, we find that the bulge velocity ellipsoid is nearly isotropic, supporting the Minniti et al. (1992) conclusion that the Galactic bulge is flattened by rotation and not by velocity anisotropies. The shape of the velocity ellipsoid of the Galactic bulge is different than that in the other components of the Galaxy (thin disk, thick disk and halo), that have been measured locally. There are trends of the kinematics with metallicity, in the sense that the metal rich subsamples have larger rotation and smaller velocity dispersions. A very important byproduct of a larger proper motion study in the bulge will be the determination of an accurate distance to the Galactic center $R_{0}$. This distance scale is absolutely essential to determine the age of the Galactic bulge: for $\mathrm{R}_{0}=$ 7 and $8.5 \mathrm{kpc}$, population models give a difference in ages of $4 \mathrm{Gyr}$, the bulge being younger for the larger $R_{0}$.
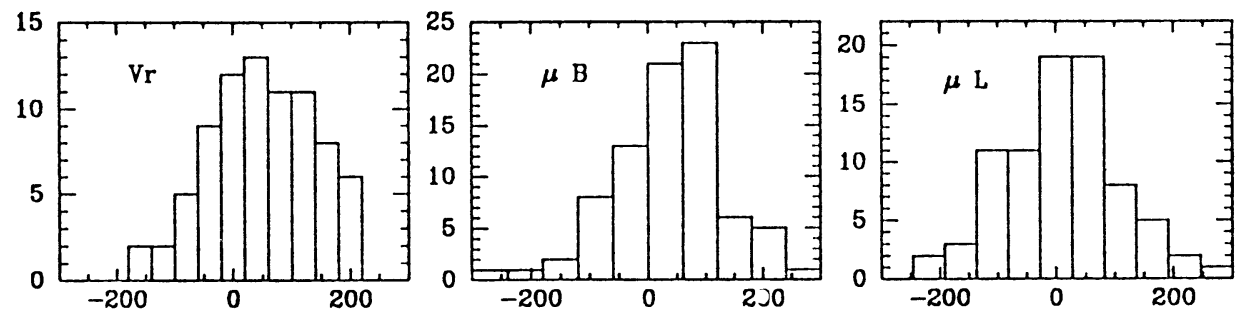